\title{
Treatment of patients with geriatric depression with repetitive transcranial magnetic stimulation
}

\author{
F. Leblhuber ${ }^{1} \cdot$ K. Steiner ${ }^{1} \cdot$ Dietmar Fuchs $^{2} \mathbb{D}$
}

Received: 8 February 2019 / Accepted: 20 June 2019 / Published online: 27 June 2019

(c) The Author(s) 2019

\begin{abstract}
Repetitive transcranial magnetic stimulation (rTMS) has become a useful tool to treat different neuropsychiatric conditions such as depression, dementia and extrapyramidal syndromes insufficiently responding to conventional treatment. In this SHAM-controlled exploratory study safety, symptom improvement as well as changes in inflammation markers and neurotransmitter precursor amino acids availability were studied after a prefrontal cortex (PFC) stimulation using rTMS as addon treatment in 29 patients with geriatric depression. Out of these, ten patients received SHAM treatment. Treatment was well tolerated, no serious adverse effects were observed. A clear improvement in symptoms of depression with a significant decrease in the HAMD-7 $(U=3.306, p=0.001)$ was found by rTMS treatment. In parallel, serum phenylalanine dropped significantly $(U=2.340, p<0.02)$, and there was a decline of tryptophan and of Phe/Tyr concentrations, both the effects, however, failed to reach the levels of statistical significance. In the patients who underwent SHAM treatment, no significant changes of HAMD-7 or the concentrations of any biomarker in the study could be found. In addition to the significant effect of rTMS on depression scores, these results point to a possible influence of rTMS on the enzyme phenylalanine hydroxylase $(\mathrm{PAH})$, which plays a crucial role in the biosynthesis of neurotransmitter precursors related to geriatric depression.
\end{abstract}

Keywords Geriatric depression - Transcranial magnetic stimulation · Phenylalanine hydroxylase · Tryptophan metabolism · Neopterin

\section{Introduction}

Repetitive transcranial magnetic stimulation (rTMS) is a promising treatment for depressive disorders, delivering focused magnetic field pulses to the brain, it can influence brain function if delivered repetitively. The magnetic field induced by rTMS can excite or inhibit a small brain area, thus altering cortical excitability (Hallet 2007; Noda et al. 2015). Previous studies described rTMS as effective

Electronic supplementary material The online version of this article (https://doi.org/10.1007/s00702-019-02037-5) contains supplementary material, which is available to authorized users.

Dietmar Fuchs

dietmar.fuchs@i-med.ac.at

1 Department of Gerontology, Kepler University Clinic, Linz, Austria

2 Division of Biological Chemistry, Biocenter, Innsbruck Medical University, Innrain 80, 4th Floor, Room M04-313, 6020 Innsbruck, Austria intervention in depressive illness, especially as augmentation in treatment-resistant cases (Liu et al. 2014, 2017).

Currently electroconvulsive therapy (ECT) is considered as the most effective treatment for depressive disorders, but this method involves the administration of anaesthetics and muscle relaxants to avoid convulsions. rTMS does not involve a seizure, and this procedure is associated with minimal side-effects (Koren et al. 2001; Bakker et al. 2015). rTMS over the prefrontal cortex (PFC) regulates the processing of emotion and mood (Liu et al. 2017), but wide-ranging brain regions play distinct roles in the pathophysiology of this affective disorder (Dalgleish 2004; Pessoa 2017). Earlier data indicate lateralisation of emotional processing, whereby the right hemisphere predominantly processing negative and the left hemisphere processing positive affects (Prete et al. 2015). Furthermore, new potential targets for rTMS in controlling emotional processing in depression are described (Downar and Daskalakis 2013) such as the dorsomedial prefrontal cortex (DMPFC), frontopolar cortex (FPC), ventromedial prefrontal cortex (VMPFC) and ventrolateral prefrontal cortex (VLPFC). 
One of the biological risk factors of late-life depression is immune activation (Dantzer et al. 2002; Widner et al. 2002; Tiermeier 2013; Capuron et al. 2011). Chronic low-grade inflammation in ageing is associated with alterations of tryptophan and tyrosine metabolism (Capuron et al. 2011). On the one hand, the pro-inflammatory cytokine interferon- $\gamma$ stimulates the biosynthesis of tetrahydrobiopterin (BH4), which is rate-limiting for the biosynthesis of the neurotransmitters serotonin, dopamine, adrenaline and noradrenaline (Neurauter et al. 2008). On the other hand, interferon- $\gamma$ triggers the high output of reactive oxygen species in macrophages, which destroys the oxidation-labile $\mathrm{BH} 4$. Recent data suggest that oxidative loss of $\mathrm{BH} 4$ triggered by interferon- $\gamma$ can reduce the biosynthesis of catecholamines, which may relate to disturbed neurotransmitter pathways in depression (Neurauter et al. 2008; Sperner-Unterweger et al. 2014). In this exploratory intervention study, the effect of rTMS on depression scores and on serum concentrations of immune system biomarker neopterin and neurotransmitter precursor amino acids correlated to late-life depression was investigated.

\section{Patients and methods}

From 55 consecutive outpatients from the Department of Gerontology of the Neuromed Campus at the Kepler University Clinic Upper Austria with different neuropsychiatric symptomatology (somatoform, depressive and anxiety disorders, addiction, paranoia and delirious syndromes, progressive cognitive decline and dementia), 29 patients (aged mean \pm SEM: $72.4 \pm 2.10$ years, 16 females) symptoms of treatment-resistant depression (incomplete remission of depressive symptoms after adequate antidepressant treatment, Thase 2011) were included in this study. On weekdays, they underwent 10 subsequent active $(n=19$ : $71.9 \pm 2.92$ years $)$ or SHAM $(n=10: 73.3 \pm 2.69$ years $)$ rTMS stimulations of the PFC bilaterally with a magnetic loop of a Theracell ${ }^{\circledR}$ apparatus (Guth Meditec, Salach, Germany; frequency $3 \mathrm{~Hz}, 0.08 \mathrm{~T}$, duration of treatment $30 \mathrm{~min}$ ) in a randomised order at a ratio of 2:1. Stimulation of the PFC was adjusting the intensity above the individual motor threshold to elicit visible bilateral contractions of the mimic musculature in the verum group (Vamava et al. 2011). SHAM treatment was performed setting the magnetic loop of the Theracell apparatus to $3 \mathrm{~Hz}, 0.00$ Tesla, duration of treatment was $30 \mathrm{~min}$.

All patients were investigated as described earlier including routine laboratory tests and cerebral magnetic resonance tomography (MRT) to exclude circumscript cerebral lesions (Leblhuber et al. 2018). Clinical assessment was performed before and after intervention, using the 7-item Hamilton depression scale (HAMD-7; McIntyre et al. 2005).
Medication including antidepressants was given constantly throughout rTMS treatment. Patients included did not receive any psychotherapy during this intervention.

The following parameters were controlled before and after rTMS treatment: serum concentrations of neopterin, tryptophan and kynurenine, calculating the kynurenine to tryptophan ratio (Kyn/Trp) as an index of tryptophan breakdown (Widner et al. 1997; Leblhuber et al. 2018), and tyrosine and phenylalanine, calculating the phenylalanine to tyrosine ratio (Phe/Tyr) as an index of phenylalanine hydroxylase (PAH) activity (Neurauter et al. 2013). Measurements were performed immediately before first/after last rTMS treatment and thus not in a fasted state. Waist circumference was within 90 and $105 \mathrm{~cm}$ (males and females), there were neither clinical nor laboratory signs of malnutrition.

Data were analysed by the Statistical Package for Social Science (version 19, SPSS, Chicago, IL, USA) as described earlier (Leblhuber et al. 2018). To take into account that not all collected data followed a normal distribution, nonparametric Friedman and Wilcoxon signed-rank test were applied. To test for associations between variables, Spearman rank correlation analysis was performed. ANOVA with repeated measurements was applied, as between-grouping factors "group" VERUM (patients) vs. SHAM (controls) were chosen, as within-grouping factor "phase" time points before and after treatment. In each ANOVA, number of observation was 58 . For the overall models, there were 30 degrees of freedom, which were divided into 1 degree of freedom each for "treatment", "time" and the interaction term "time\#treatment"; the remaining 27 degrees of freedom being associated with the residual error. Calculations were performed with statistical package "STATA", version 14.2 (StataCorp LLC, 4905 Lakeway Drive, College Station, Texas 77845 , USA). $p$ values below 0.05 were considered to indicate significance.

The study was approved by the local ethics committee. Patients were treated with rTMS after informed consent according to the declaration of Helsinki.

\section{Results}

Routine laboratory tests including leucocyte count and C-reactive protein showed results within normal limits. rTMS intensity is well-tolerated without any serious adverse events (Vamava et al. 2011). rTMS induced significant depression symptom improvement and a significant decrease in the HAMD-7 score after active treatment (mean \pm SEM, before $12.9 \pm 0.89$, after: $10.2 \pm 0.67 ; U=3.306, p=0.001$, Fig. 1). No effect was found in the SHAM-treated group (13.2 \pm 1.43 before, after: $13.3 \pm 1.48 ; U=0.447$, n.s.).

In the rTMS-treated group $(n=19)$, average concentration of tryptophan before intervention was 


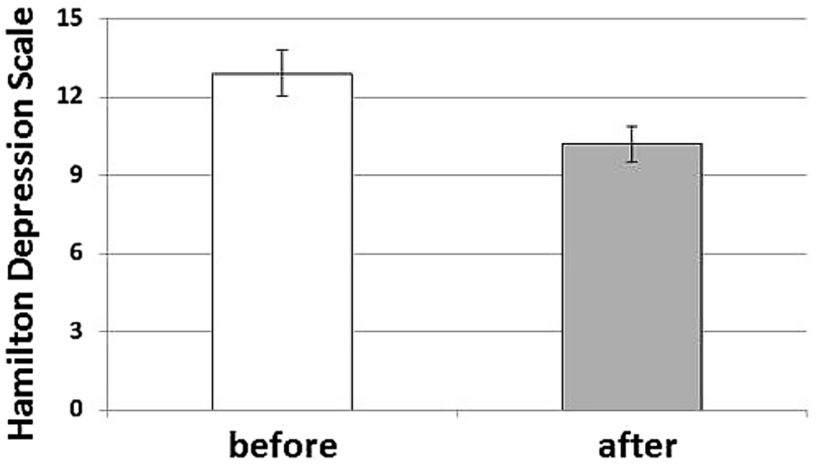

Fig. 1 HAMD-7 depression scale in 19 patients with geriatric depression before and after 10 sessions of rTMS treatment (mean values \pm SEM are shown; $U=3.306 ; p<0.001$ )

$58.6 \pm 3.56 \mathrm{nmol} / \mathrm{L}$, of kynurenine $1.85 \pm 0.10 \mu \mathrm{mol} / \mathrm{L}$, of $\mathrm{Kyn} / \mathrm{Trp}, 33.3 \pm 2.53 \mu \mathrm{mol} / \mathrm{mmol}$, of tyrosine $83.9 \pm 5.82 \mu \mathrm{mol} / \mathrm{L}$, of phenylalanine $98.2 \pm 7.22 \mu \mathrm{mol} / \mathrm{L}$, and of Phe/Tyr was $1.19 \pm 0.051 \mu \mathrm{mol} / \mu \mathrm{mol}$. The average neopterin concentration was $10.6 \pm 0.79 \mathrm{nmol} / \mathrm{L}$ and nitrite concentration was $45.8 \pm 7.65 \mu \mathrm{mol} / \mathrm{L}$ (see Table 1 ).

Upon rTMS treatment, the phenylalanine concentrations changed significantly, they decreased to $81.4 \pm 4.61 \mu \mathrm{mol} / \mathrm{L}(U=2.340, p<0.02)$, concentrations of all other biomarkers did not change significantly.

Correlations existed between serum concentrations of neopterin and $\mathrm{Kyn} / \mathrm{Trp}(\mathrm{rs}=0.441, p<0.02$ before $\mathrm{rTMS})$, results similar to earlier measurements in larger populations, indicating immune activation in late-life depression as described in patients with $\mathrm{AD}$ and other forms of dementia (Widner et al. 2000).

After ten sessions of rTMS treatment, measurements of biomarkers were repeated (Table 1), and phenylalanine concentrations declined significantly $(p<0.02$, see Fig. 2$)$.

Table 1 Serum concentrations (mean values \pm SEM) of neopterin, nitrite and neurotransmitter precursor amino acids in 19 patients with late-life depression before and after rTMS treatment

\begin{tabular}{lllll}
\hline & Before rTMS & After rTMS & $U$ & $p$ \\
\hline Tryptophan $(\mu \mathrm{mol} / \mathrm{L})$ & $58.6 \pm 3.56$ & $52.5 \pm 2.53$ & 0.092 & n.s \\
Kynurenine $(\mu \mathrm{mol} / \mathrm{L})$ & $1.85 \pm 0.10$ & $1.92 \pm 0.13$ & 0.275 & n.s \\
Kyn/Trp $(\mu \mathrm{mol} / \mathrm{mmol})$ & $33.3 \pm 2.53$ & $37.2 \pm 2.61$ & 0.046 & n.s \\
Tyrosine $(\mu \mathrm{mol} / \mathrm{L})$ & $83.9 \pm 5.82$ & $73.6 \pm 5.70$ & 0.459 & n.s \\
Phenylalanine $(\mu \mathrm{mol} / \mathrm{L})$ & $98.2 \pm 7.22$ & $81.4 \pm 4.61$ & 2.340 & $<0.02$ \\
Phe/Tyr $(\mu \mathrm{mol} / \mu \mathrm{mol})$ & $1.19 \pm 0.051$ & $1.15 \pm 0.061$ & 1.516 & n.s \\
Nitrite $(\mu \mathrm{mol} / \mathrm{L})$ & $45.8 \pm 7.65$ & $40.3 \pm 11.4$ & 0.872 & n.s \\
Neopterin $(\mathrm{nmol} / \mathrm{L})$ & $10.6 \pm 3.43$ & $11.7 \pm 1.24$ & 1.423 & n.s \\
\hline
\end{tabular}

Kyn/Trp kynurenine to tryptophan ratio, Phe/Tyr phenylalanine to tyrosine ratio

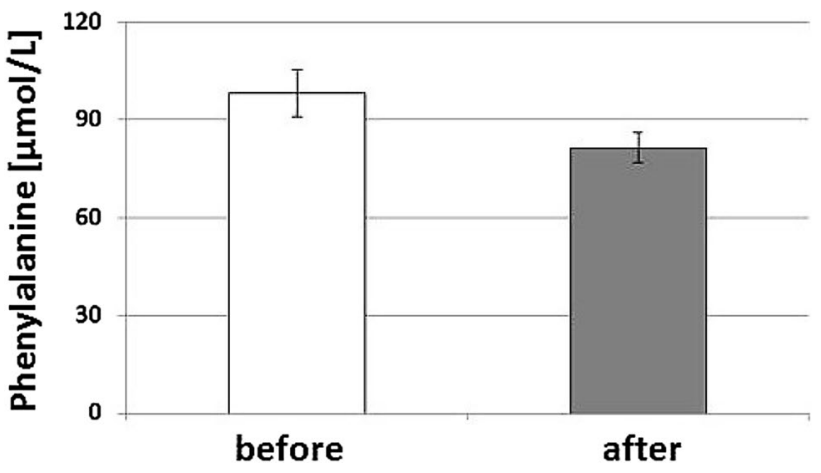

Fig. 2 Serum phenylalanine concentrations in 19 patients with geriatric depression before and after a series of ten rTMS sessions (mean values \pm SEM are shown; $U=2.340, p<0.02$ )

No significant effect was observed for any other measurement in this study (Table 1). There were also no significant effects in the SHAM group.

ANOVA with repeated measurements was designed as follows: as between subjects-variable "treatment" (i.e. verum $v s$. sham) was chosen, and as within subjects-variable, "time" (before versus after) was used. For phenylalanine, "treatment" $(F=8.85,1 d f, p=0.0061)$ and "time" $(F=4.28,1 d f, p=0.0483)$ were significant; the interaction term was insignificant $(F=0.36,1 d f, p=0.55)$. For Phe/ Tyr, only "treatment" $(F=4.55,1 d f, p=0.422)$ was significant; "time" $(F=0.57,1 d f, p=0.4560)$ and the interaction term $(F=0.02,1 d f, p=0.8902)$ failed to reach significance. Finally, for HAM scores, "treatment" $(F=1.40$, $1 d f, p=0.2464)$ was not significant in contrast to "time" $(F=7.52,1 d f, p=0.0107)$; here, also a significant interaction term was found $(F=8.73,1 d f, p=0.0064)$.

Post hoc tests: Fig. 3 explains this behaviour of HAM scores [all $p$ values obtained by paired (red) or unpaired (black) Student's $t$ tests]: in patients, the difference between pre- and post-treatment values is highly significant $(p=0.0008)$, while in controls there is practically no effect of treatment $(p=0.68)$. The difference between verum and sham pre-treatment values is not significant $(p=0.85)$; due to the significant treatment effect in patients, however, the post-treatment values differ between verum and sham $(p=0.0371)$.

\section{Discussion}

rTMS is an evidence-based noninvasive treatment for depression (Shin et al. 2016; Liu et al. 2017) approved by the US Food and Drug Administration (FDA, Blumberger et al. 2018), which gave also promising results in the treatment of other neuropsychiatric disorders, such as Parkinson's disease (von Papen et al. 2014; Dagan et al. 2017), essential tremor 


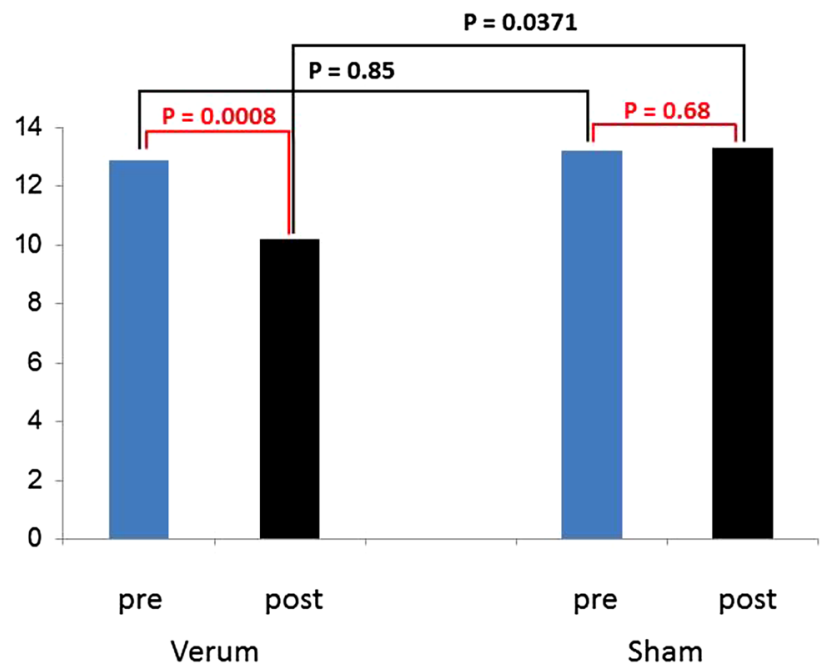

Fig. 3 Post hoc tests: behaviour of HAM scores [all $p$ values obtained by paired (red) or unpaired (black) Student's $t$ tests] in patients: the difference between pre- and post-treatment values is highly significant $(p=0.0008)$, while in controls there is practically no effect of treatment $(p=0.68)$. The difference between verum and sham pretreatment values is not significant $(p=0.85)$; due to the significant treatment effect in patients, however, the post-treatment values differ between verum and sham $(p=0.0371)$

(Kang and Cauraugh 2017), stroke (Watanabe et al. 2018), cognitive decline and dementia (Rabey and Dobronevsky 2016; Koch et al. 2017).

Depression is a leading cause of disability worldwide contributing substantially to the global disease burden (Murray et al. 2012; Otte et al. 2016). A major challenge in treating geriatric depression is the lack of robust efficacy for many treatments that are of significant benefit to depressed working age adults (Sabesan et al. 2015).

Significant alterations of neurotransmitter levels have been previously reported in depressive syndromes (Price et al. 2009). Pro-inflammatory cytokines upregulate the biosynthesis of BH4 (Haruki et al. 2016), which is essential for the biosynthesis of serotonergic as well as adrenergic neurotransmitters a rate-limiting cofactor (Sperner-Unterweger et al. 2014).

Chronic immune activation, characteristic for depressive syndromes including geriatric depression-also seen in the herein-reported study - upregulates the production of reactive oxygen species (ROS) (Widner et al. 2000) in macrophages and destroys the oxygen labile BH4. This may lead to a reduced biosynthesis of serotonin, dopamine, adrenaline and noradrenaline, all of them important in the pathophysiology of depression (Neurauter et al. 2008; Sperner-Unterweger et al. 2014).
rTMS is an evidence-based treatment for major depressive disorder not responding to pharmacotherapy (Blumberger et al. 2018). Emerging data show positive results of rTMS in refractory geriatric depression. The evidence regarding safety as well as efficacy of rTMS in geriatric depression were discussed earlier (Galvez et al. 2015; Sabesan et al. 2015).

This exploratory intervention study further describes evidence regarding the safety and efficacy of rTMS in 19 patients with late-life depression. Patients were treated with ten consecutive sessions of rTMS within 2 weeks: rTMS induced a significant depression symptom improvement with a significant decrease in the HAMD-7 scale $(p=0.001)$ compared to 10 SHAM-treated patients. The phenylalanine concentrations simultaneously declined $(p<0.02)$ similar to earlier findings in patients with severe depression responding to electroconvulsive therapy.

Our still preliminary finding could relate to a central role of the BH4 activity in the pathophysiology of depression (Anderson et al. 1994) and may indicate that rTMS influences the enzyme PAH. PAH plays a key role in the biosynthesis of neurotransmitters noradrenaline and adrenaline which are down-stream products of tyrosine. Notably, Phe/Tyr concentrations, an index of PAH activity was not significantly influenced by rTMS. Thus, there might be another background for the decrease of phenylalanine: it could possibly relate to a change of the nutritional behaviour of patients because phenylalanine is an essential amino acid, but interestingly the concentrations of another essential amino acid, namely tryptophan, did not change significantly. Thus, it is still unclear by which mechanism rTMS contributes to the decrease of phenylalanine levels, still most probably by a functional improvement of PAH.

There are limiting factors for interpretation of this exploratory pilot study. First, only 19 patients with geriatric depression were included and treated with rTMS. Beyond that, only correlational evidence between neurotransmitter changes and noninvasive rTMS could be shown, a causative relationship still has to be verified in larger double blind SHAM controlled studies. Second, the decline of concentrations of essential amino acid phenylalanine occurred independent of any noticeable change of biomarkers of immune activation, because concentrations of neopterin and Kyn/Trp remained unchanged.

Acknowledgements Open access funding provided by University of Innsbruck and Medical University of Innsbruck. The authors wish to thank Prof. Gilbert Reibnegger from the Medical University of Graz, Austria for his support with the statistical analyses.

\section{Compliance with ethical standards}

Conflict of interest The authors declare that they have no competing interest. 
Open Access This article is distributed under the terms of the Creative Commons Attribution 4.0 International License (http://creativeco mmons.org/licenses/by/4.0/), which permits unrestricted use, distribution, and reproduction in any medium, provided you give appropriate credit to the original author(s) and the source, provide a link to the Creative Commons license, and indicate if changes were made.

\section{References}

Anderson DN, Wilkinson AM, Abou-Saleh MT, Blair JA (1994) Recovery from depression after electroconvulsive therapy is accompanied by evidence of increased tetrahydrobiopterindependent hydroxylation. Acta Psychiatr Scand 90:10-13

Bakker N, Shahab S, Giacobbe P, Blumberger DM, Daskalakis ZJ, Kennedy SH, Downar J (2015) rTMS of the dorsomedial prefrontal cortex for major depression: safety, tolerability, effectiveness, and outcome predictors for $10 \mathrm{~Hz}$ versus intermittent theta-burst stimulation. Brain Stimul 8:208-215

Blumberger DM, Vila-Rodriguez F, Thorpe KE, Feffer K, Noda Y, Giacobbe P, Knyahnytska Y, Kennedy SH, Lam RW, Daskalakis ZJ, Downar J (2018) The effectiveness of theta burst versus highfrequency repetitive trancranial magnetic stimulation in patients with depression (THREE-D): a randomised non-inferiority trial. Lancet 391:1683-1692

Capuron L, Schroecksnadel S, Féart C, Aubert A, Higueret D, Barberger-Gateau P, Layé S, Fuchs D (2011) Chronic low grade immune activation in the elderly is associated with increased tryptophan catabolism and altered phenylalanine turnover: role in neuropsychiatric symptomatology. Biol Psychiatry 70:175-182

Dagan M, Hermann T, Mirelman A, Giladi N, Hausdorff JM (2017) The role of the prefrontal cortex in freezing of gait in Parkinson's disease: insights from a deep repetitive transcranial magnetic stimulation exploratory study. Exp Brain Res 235:2463-2472

Dalgleish T (2004) The emotional brain. Nat Rev Neurosci 5:583-589

Dantzer R, Wollman EE, Yirmiya R (2002) Cytokines and depression: an update. Brain Behav Immun 16:501-502

Downar J, Daskalakis ZJ (2013) New targets for rTMS in depression: a review of convergent evidence. Brain Stimul 6:231-240

Galvez V, Ho KA, Alonzo A, Martin D, George D, Loo CK (2015) Neuromodulation therapies for geriatric depression. Curr Psychiatry Rep 17(7):59. https://doi.org/10.1007/s11920-015-0592-y

Hallet M (2007) Transcranial magnetic stimulation, a primer. Neuron 55(2):187-199

Haruki H, Hovius R, Pedersen MG, Johnsson K (2016) Tetrahydrobiopterin biosynthesis as a potential target of the kyurenine pathway metabolite xanthurenic acid. J Biol Chem 291(2):652-657

Kang N, Cauraugh JH (2017) Does non-invasive brain stimulation reduce essential tremor? A systemic review and meta-analysis. PLoS ONE 12(9):e0185462. https://doi.org/10.1371/journ al.pone.0185462(eCollection2017)

Koch G, Bonni S, Pellicciari MC, Casula EP, Mancini M, Esposito R, Ponzo V, Picazio S, Di Lorenzo F, Serra L, Motta C, Maiella M, Marra C, Cercignani M, Martorana A, Caltagirone C, Bozzali M (2017) Transcranial magnetic stimulation of the precuneus enhances memory and neural activity in prodromal Alzheimer's disease. Neuroimage 169:302-311

Koren D, Shefer O, Chistyakov A, Kaplan B, Feinsod M, Klein E (2001) Neuropsychological effects of prefrontal slow rTMS in normal volunteers: a double-blind sham-controlled study. $\mathrm{J}$ Clin Exp Neuropsychol 23(4):424-430. https://doi.org/10.1076/ jcen.23.4.424.1225

Leblhuber F, Steiner K, Schuetz B, Fuchs D, Gostner JM (2018) Probiotic supplementation in patients with Alzheimer's dementia - an exploratory intervention study. Curr Alzheimer Res 15(12):1106-1113

Liu B, Zhang Y, Zhang L, Li L (2014) Repetitive transcranial magnetic stimulation as an augmentative strategy for treatment resistant depression, a meta-analysis of randomised, double-blind and sham-controlled study. BMC Psychiatry 14:342-350

Liu S, Sheng J, Li B, Zhang X (2017) Recent advances in non-invasive brain stimulation for major depressive disorder. Front Hum Neurosci 11:526

McIntyre RS, Konarski JZ, Mancini DA, Fulton KA, Parikh SV, Grigoriadis S, Grupp LA, Bakish D, Filteau MJ, Gorman C, Nemeroff CB (2005) Kennedy SH (2005) Measuring the severity of depression and remission in primary care: validation of the HAMD-7 scale. CMAJ 173(11):1327-1334

Murray CJ et al (2012) Disability-adjusted life years (DALYs) for 291 diseases and injuries in 21 regions, 1990-2010: a systematic analysis for the Global Burden of Disease Study 2010. Lancet 380:2197-2223

Neurauter G, Schröcksnadel K, Scholl-Bürgi S, Sperner-Unterweger B, Schubert C, Ledochowski M, Fuchs D (2008) Chronic immune stimulation correlates with reduced phenylalanine turnover. Curr Drug Metab 9(7):622-627

Neurauter G, Scholl-Buergi S, Haara A, Geisler S, Mayersbach P, Schennach H, Fuchs D (2013) Simultaneous measurement of phenylalanine and tyrosine by high performance liquid chromatography (HPLC) with fluorescence detection. Clin Biochem 46:1848-1851

Noda Y, Silverstein WK, Barr MS, Vila-Rodriguez F, Downar J, Rajji TK, Fitzgerald PB, Mulsant BH, Vigod SN, Daskalakis ZJ, Blumberger DM (2015) Neurobiological mechanisms of repetitive transcranial magnetic stimulation of the dorsolateral prefrontal cortex in depression: a systemic review. Psychol Med 45:3411-3432

Otte C, Gold SM, Penninx BW, Pariante CM, Etkin A, Fava M, Mohr DC, Schatzberg AF (2016) Major depressive disorder. Nat Rev Dis Primer 2:16065. https://doi.org/10.1038/nrdp.2016.65

Pessoa L (2017) A network model of the emotional brain. Trends Cogn Sci 21:357-371

Prete G, Laeng B, Fabri M, Foschi N, Tommasi L (2015) Right hemisphere or valence hypothesis, or both? The processing of hybrid faces in the intact callosotomized brain. Neuropsychologia 68:94-106

Price RB, Shungu DC, Mao X, Nestadt P, Kelly C, Collins KA, Murrough JW, Charney DS, Mathew SJ (2009) Amino acid neurotransmitters assessed by H MRS: relationship to treatment-resistance in major depressive disorder. Biol Psychiatry 65(9):792-800

Rabey JM, Dobronevsky E (2016) Repetitive transcranial magnetic stimulation (rTMS) combined with cognitive training is a safe and effective modality for the treatment of Alzheimer's disease: clinical experience. J Neural Transm (Vienna) 123(12):1449-1455

Sabesan P, Lankappa S, Khalifa N, Krishnan V, Gandhi R, Palaniyappan L (2015) Transcranial magnetic stimulation for geriatric depression: promises and pitfalls. World J Psychiatry 5(2):170-181

Shin HW, Youn YC, Chung SJ, Sohn YH (2016) Effect of high-frequency repetitive transcranial magnetic stimulation on major depressive disorder in patients with Parkinson's disease. J Neurol 263(7):1442-1448

Sperner-Unterweger B, Kohl C, Fuchs D (2014) Immune changes and neurotransmitters: possible interactions in depression? Prog Neuropsychopharmacol Biol Psychiatry 48:268-276

Thase ME (2011) Treatment-resistant depression: prevalence, risk factors, and treatment strategies. J Clin Psychiatry 72(5):e18. https:// doi.org/10.4088/JCP.8133tx4c

Tiermeier H (2013) Biological risk factors for late life depression. Eur J Epidemiol 18(8):745-750 
Vamava A, Stokes MG, Chambers CD (2011) Reliability of the "observation of movement" method for determining motor threshold using transcranial magnetic stimulation. J Neurosci Methods 201(2):327-332

von Papen M, Fisse M, Sarfeld AS, Fink GR, Nowak DA (2014) The effects of $1 \mathrm{~Hz}$ rTMS preconditioned by tDCS on gait kinematics in Parkinson's disease. J Neural Transm (Vienna) 12(7):743-754

Watanabe K, Kudo Y, Sugawara E, Nakamizo T, Amari K, Takahashi K, Tanaka O, Endo M, Hayakawa Y, Johkura K (2018) Comparative study of ipsilateral and contralateral magnetic stimulations for acute infarction. J Neurol Sci 384:10-14

Widner B, Werner ER, Schennach H, Wachter H, Fuchs D (1997) Simultaneous measurements of serum tryptophan and kynurenine by HPLC. Clin Chem 43:2424-2426
Widner B, Leblhuber F, Walli J, Tilz GP, Demel U, Fuchs D (2000) Tryptophan degradation and immune activation in Alzheimer's disease. J Neural Transm (Vienna) 107:343-353

Widner B, Laich A, Sperner-Unterweger B, Ledochowski M, Fuchs D (2002) Neopterin production tryptophan degradation and mental depression: what is the link? Brain Behav Immun 16:590-595

Publisher's Note Springer Nature remains neutral with regard to jurisdictional claims in published maps and institutional affiliations. 